\title{
Comparison of intraspinal and intrathecal implantation of induced pluripotent stem cell-derived neural precursors for the treatment of spinal cord injury in rats
}

Takashi Amemori ${ }^{1}$, Jiri Ruzicka ${ }^{1,2}$, Nataliya Romanyuk' ${ }^{1}$, Meena Jhanwar-Uniyal ${ }^{3}$, Eva Sykova ${ }^{1,2}$ and Pavla Jendelova ${ }^{1,2^{*}}$

\begin{abstract}
Background: Stem cell treatment provides a promising therapy for patients with spinal cord injury (SCl). However, the applied stem cells exert their effects in different manners that are dependent on the route used for administration.

Methods: In the present study, we administered neural precursors derived from induced pluripotent stem cells (iPS-NPs) either intraspinally into the lesion center or intrathecally into the subarachnoid space of rats with a ballooninduced spinal cord compression lesion. Functional locomotor performance, cell survival, astrogliosis, axonal sprouting and the expression of endogenous neurotrophic growth factors were evaluated using behavioral tests (BBB, flat beam test, rotarod, plantar test), morphometric analysis, immunohistochemistry and qPCR.

Results: Both treatments facilitated the functional locomotor recovery of rats with SCI. iPS-NPs injected intraspinally survived well for 2 months and were positive for MAP2, while cells grafted intrathecally were undetectable at the site of administration or in the spinal cord tissue. Intraspinal implantation increased gray and white matter sparing and axonal sprouting and reduced astrogliosis, while intrathecal application resulted only in an improvement of white matter sparing and an increase in axonal sprouting, in parallel with no positive effect on the expression of endogenous neurotrophic growth factor genes or glial scar reduction.

Conclusions: Intrathecally grafted iPS-NPs had a moderate therapeutic benefit on SCI through a paracrine mechanism that does not require the cells to be present in the tissue; however, the extended survival of i.s. grafted cells in the spinal cord may promote long-term spinal cord tissue regeneration.
\end{abstract}

Keywords: Spinal cord injury, Human induced pluripotent stem cells, Cell therapy, Cell application route, Neural progenitors, Intraspinal injection, Intrathecal injection

\section{Background}

The National Spinal Cord Injury Statistical Center has reported that approximately $66 \%$ of human spinal cord injury (SCI) cases have incomplete lesions caused by vehicular accidents, falls, violence, sports, etc. (https:// www.nscisc.uab.edu/reports.aspx). Successful treatment of

\footnotetext{
* Correspondence: jendel@biomed.cas.cz

${ }^{1}$ Department of Neuroscience, Institute of Experimental Medicine, Academy of Sciences of the Czech Republic, Videnska 1083, 14220 Prague 4, Czech Republic

${ }^{2}$ Department of Neuroscience, 2nd Faculty of Medicine, Charles University, Plzenska 130/221, 15000 Prague 5, Czech Republic

Full list of author information is available at the end of the article
}

SCI is difficult owing to the limited regeneration of nervous tissue and its inability to replace lost neurons and injured axons. Different types of stem cells, including embryonic, fetal, and adult stem cells, have been transplanted into animal models of SCI. Cell grafting as a therapeutic strategy for the treatment of SCI is promising, since it can target cell replacement, neuroprotection, and regeneration.

In particular, the use of induced pluripotent stem cells (iPS) is in the center of attention, since these cells can be tailored to patients for autologous use to avoid immune rejection, ethical constraints, and tissue donation 
[1-4]. Several studies have evaluated the efficacy of iPSderived neural precursor cells (iPS-NPs) in animal models of SCI (recently reviewed in $[3,5]$ ). When injected into SCI, these cells differentiated predominantly into glia or neurons, formed synapses with host axons and increased regeneration, leading to functional improvement [6-10]. Moreover, specific cell types, such as oligodendrocyte precursors, can be obtained from iPS, which after transplantation can remyelinate host axons following SCI [11, 12]. However, iPS-NPs can also reduce secondary damage through immunomodulation and neurotrophic effects. Human iPS-NPs can spare tissue in the lesion area [7-9], produce cytokines and neurotrophic factors such as neurotrophin 4 (NT4), glia-derived neurotrophic factor (GDNF), and interleukin (IL)-10 [13], and stimulate angiogenesis via the production of vascular endothelial growth factor (VEGF) [7].

In all studies on SCI so far reported, iPS were implanted directly into the spinal cord tissue. However, the direct injection of stem cells into the spinal cord may cause additional damage resulting in further deterioration of the lesioned tissue. In addition, the risk of tumor or teratoma formation is still not negligible, since nestin-positive tumor formation was observed 103 days after the transplantation of iPS-derived neurospheres into a mouse model of SCI. Similarly, teratomas were found after the grafting of murine iPS-derived neurospheres into contusive SCI [14]. On the other hand, the intrathecal (i.t.) application of cells can overcome some of these limitations. Moreover, several studies have shown that intrathecally administered stem cells can partially home into the tissue parenchyma [15-17] while exerting positive therapeutic effects on SCI, even without their long-term survival [18].

Our previous studies have shown that the direct injection of mesenchymal stem cells (MSCs) and/or olfactory ensheathing glia cells as well as adipose-derived MSCs $[19,20]$, human fetal neural stem cells derived from the spinal cord [21], and human iPS-NPs [9] into a balloon-induced spinal cord compression lesion promotes locomotor recovery. Among all of the cell types used in those previous studies, the application of iPS-NPs resulted in the fastest and most pronounced locomotor recovery accompanied by a high degree of tissue regeneration in a rat model of SCI [9]. iPS-NPs also had a beneficial effect in a rat stroke model [22]. In both studies with iPS-NPs [9, 22], the effect on functional recovery was rapid and preceded grafted cell differentiation and maturation. We therefore assume that a paracrine effect plays an important role in spinal cord regeneration. In the present study, we injected iPS-NPs intrathecally 1 week after SCI, when the hostile environment created by the inflammatory response following SCI is diminished [23, 24]. An intraspinal (i.s.) injection of iPS-NPs into the lesion center at the same time point was used for comparison as a positive control. Behavioral outcome, morphological changes, the expression of neurotrophic factor genes, and axonal regeneration were examined 8 weeks after transplantation (9 weeks after SCI). As a model of SCI, we chose a balloon-induced spinal cord compression lesion, which simulates the pathology caused by the compression of the human spinal cord by an unreduced dislocation or a fracture dislocation of the spine [25]. It is assumed that both mechanical and vascular factors are involved in the pathogenesis of SCI in this model. The balloon compression model is simple and reproducible, and unlike other models (clip injury, weight drop injury) does not require a laminectomy.

\section{Methods}

\section{Cell preparation}

Clone selection, validation of the iPS line, and the derivation of neuronal precursors have been described in detail by Polentes et al. [22]. Briefly, the human iPS line was derived from female (IMR90) human fetal lung fibroblasts (ATCC, Teddington, UK) and transduced with a lentivirus-mediated combination of OCT4, SOX2, NANOG, and LIN28 human cDNA [22]. Early neural precursors were produced in low-attachment culture in the presence of Noggin $(500 \mathrm{ng} / \mathrm{ml}$; R\&D Systems, Minneapolis, MN, USA), the transforming growth factor-beta pathway inhibitor SB 431542 (10 nM; Sigma, St. Louis, MO, USA), and basic fibroblast growth factor (bFGF; $10 \mu \mathrm{g} / \mathrm{ml}$ ) and brain-derived neurotrophic factor (BDNF; $20 \mu \mathrm{g} / \mathrm{ml}$ ) (both Pepro Tech, London, UK). Human iPS-NPs were routinely cultured in tissue culture flasks coated with poly-L-ornithine $(0.002 \%$ in distilled water) and laminin $(10 \mu \mathrm{g} / \mathrm{ml}$ in Dulbecco's modified Eagle's medium (DMEM):F12; Sigma). Growth media were comprised of DMEM:F12 and Neurobasal medium (1:1), B27 supplement (1:50), N2 supplement (1:100) (GIBCO, Life Technologies, Grand Island, NY, USA), L-glutamine ( $2 \mathrm{mM}$; Sigma), penicillin and streptomycin (50 $\mathrm{U} / \mathrm{ml}$; GIBCO), and fibroblast growth factor (FGF; $10 \mathrm{ng} / \mathrm{ml}$ ), epidermal growth factor (EGF; $10 \mathrm{ng} / \mathrm{ml}$ ) and BDNF $(20 \mathrm{ng} / \mathrm{ml})$ (PeproTech). Prior to transplantation the iPS-NPs were predifferentiated over 7 days in the same medium except for the omission of FGF and EGF [9]. The neural precursors were characterized prior to transplantation. They were negative for the pluripotent markers nanog, SSEA4, and TRA-1-60, slightly positive for oct $3 / 4$ (30\%) and sox2 (50\%), and positive (>80 \%) for SSEA1, CD133, CD24, CD29, NCAM, nestin, and A2B5. Their full characterization, including mRNA expression, is described by Polentes et al. and Romanyuk et al. $[9,22]$. 


\section{Animals}

Adult male Wistar rats aged 10 weeks and weighing 270-300 g $(n=38)$ were obtained from our facility in the Academy of Sciences of the Czech Republic. All experiments were performed in accordance with the European Communities Council Directive of $22^{\text {nd }}$ of September $2010(2010 / 63 / \mathrm{EU})$ regarding the use of animals in research and were approved by the Ethics Committee of the Institute of Experimental Medicine, Academy of Sciences of the Czech Republic.

\section{Surgical procedure}

The animals were anesthetized with isoflurane (Forane; Abbott Laboratories, Queenborough, UK), and the spinal cord was exposed at thoracic vertebra 10. A sterile 2french Fogarty catheter was inserted into the epidural space until the center of the balloon rested on thoracic vertebra 8 (T8). The balloon was rapidly inflated with $15 \mu \mathrm{l}$ saline for 5 minutes. During this procedure, $3 \%$ isoflurane in air was administered at a flow rate of $0.3 \mathrm{l} /$ minute, and the animal's body temperature was kept at $37{ }^{\circ} \mathrm{C}$ with a heating pad. After the catheter was deflated and removed, the incised skin and muscle were sutured. The operated animals were assisted in feeding and urination until they had recovered sufficiently to perform these functions on their own. The animals received gentamicin sulfate $(5 \mathrm{mg} / \mathrm{kg})$ for 3 days to prevent postoperative infections and were allowed to feed and drink ad libitum.

\section{Transplantation}

One week after the spinal cord was lesioned at T8 by balloon compression, $5 \times 10^{5}$ human iPS-NPs (12 ${ }^{\text {th }}$ passage) in $50 \mu \mathrm{l}$ saline $(n=9)$ or $50 \mu \mathrm{l}$ saline without cells $(n=9)$ were injected intrathecally between L3 and L4 or L4 and L5 through a $25 \mathrm{G}$ needle for 30 seconds. Any remaining cells left in the needle were flushed by an additional injection of $50 \mu \mathrm{l}$ saline, and the injection needle was left in place for 30 seconds to prevent backflow of the injected content. The position of the needle tip was confirmed by the backflow of cerebrospinal fluid (CSF) into the hub. The i.s. injection of $5 \times 10^{5}$ human iPS-NPs (12 ${ }^{\text {th }}$ passage) in $5 \mu$ l saline $(n=9)$ or $5 \mu$ l saline without cells $(n=11)$ was also performed 1 week after SCI. The spinal cord was exposed at $\mathrm{T} 8$, and the injection was made in the midline of the spinal cord at a depth of $1 \mathrm{~mm}$ below the dorsal surface with an injection rate of $1 \mu \mathrm{l} /$ minute using a Nano-Injector (Stoelting CO., Wood Dale, IL, USA). The needle was kept in place for a further 5 minutes to prevent leakage of the cell suspension. Cyclosporine A $(10 \mathrm{mg} / \mathrm{kg})$, azathioprine sodium (2 mg/kg), methylprednisolone $(2 \mathrm{mg} / \mathrm{kg}$, tapered to $0.5 \mathrm{mg} / \mathrm{kg}$ ), and ampicillin $(50-100 \mathrm{mg} / \mathrm{kg}$ ) were administered 1 day before transplantation and every day throughout the experiments.

\section{Functional analysis}

Animals with and without cell treatment were trained and examined using the plantar, rotarod, and flat beam walking tests before and after surgery during a 2-month observation period. Simultaneously, the animals' locomotion was evaluated by the Basso, Beattie, and Bresnahan (BBB) scale [26] every week following SCI. The details of these behavioral tests have been published elsewhere $[9,21]$ but are briefly explained in the following.

The BBB test evaluates joint movement, paw placement, weight support, forelimb-hindlimb coordination, and other parameters using a $0-21$ point scale. The plantar test examines the sensitivity of the paw to a noxious thermal stimuli. The withdrawal latency (i.e., how quickly the animal responded to the stimulus) was measured using a Plantar Test apparatus (Ugo Basile, Comerio, Italy). Five repeated measurements were obtained from each hind paw at 5-minute intervals. In the flat beam walking test, the animal was placed at one end of a 1-m-long beam with a flat surface and oriented to walk toward the opposite end of the beam where an escape box was placed. The latency and the trajectory to traverse the beam were recorded by a video-tracking system (TSE-System Inc., Bad Homburg, Germany) for a maximum of 60 seconds. The animals were tested twice a day for three consecutive days. The locomotor performance was evaluated using a $0-7$ point scale modified from Metz and Whishaw [27], which enabled the examiner to determine how the animal could walk on the beam (e.g., by dragging their hind legs, using one or two hind legs, etc.). The rotarod test apparatus (Ugo Basile) requires a degree of motor coordination on the part of the tested animals to successfully perform the task. Each animal was placed on a rotating rod at a speed of $10 \mathrm{rpm}$ before surgery and $5 \mathrm{rpm}$ after surgery and was left to walk on the rod for 60 seconds. The animal was given four trials per day with an inter-trial interval of 5 minutes across five consecutive days. The latency to fall off the rod onto a floor plate was measured.

Data are expressed as mean \pm standard error of the mean. To compare the data between two groups, a twosample $t$ test for independent samples was performed if the two samples had equal variances. If the samples had unequal variances, the Mann-Whitney test was used for evaluation. The comparison of four groups - i.t. injection of iPS-NPs, i.s. injection of iPS-NPs, and the appropriate saline controls (i.t. and i.s.) - was performed using a two-way analysis of variance. $p<0.05$ was considered statistically significant.

\section{Histological and immunohistochemical analyses}

After completing the behavioral analyses (8 weeks after treatment, 9 weeks after SCI), the animals were deeply anesthetized with ketamine $(100 \mathrm{mg} / \mathrm{kg})$ and xylazine 
(20 $\mathrm{mg} / \mathrm{kg})$. Transcardial perfusion was performed with a phosphate buffer solution, followed by a $4 \%$ paraformaldehyde solution in phosphate buffer. A 2-cm-long segment of the spinal cord was dissected between $1 \mathrm{~cm}$ cranial and $1 \mathrm{~cm}$ caudal to the injury epicenter. Serial cross-sections were obtained with a $5 \mu \mathrm{m}$ thickness. For morphometric measurements (five i.t.-treated rats, five i.t. control rats, five i.s.-treated rats, seven i.s. control rats), six sections were selected at $1 \mathrm{~mm}$ intervals along the craniocaudal axis and stained with Luxol-Fast Blue and Cresyl Violet. Images of each cross-section were taken with an Axioskop 2 plus microscope (Carl Zeiss AG, Oberkochen, Germany) and analyzed by ImageJ software (National Institutes of Health, Bethesda, MD, USA). A series of serial sections were partly stained by antibodies against growth-associated protein (GAP43; Millipore, Billerica, MA, USA) and glial fibrillary acidic protein (GFAP; Sigma). GAP43-positive axons were manually counted. To visualize the reactivity of the anti-GFAP primary antibody, goat anti-mouse IgG conjugated with Alexa-Fluor 594 (Molecular Probes, Eugene, OR, USA) was used. Confocal images were taken with a Zeiss LSM 5 Duo confocal microscope (Car Zeiss AG). To identify human stem cells, antibodies directed against human nuclei $(\mathrm{HuNu}$; $\mathrm{Chemi-}$ con, Temecula, CA, USA) and human mitochondria (Mitochondrially Encoded Cytochrome C Oxidase II: MTCO2; Abcam, Cambridge, UK) were used. In parallel, the neuronal differentiation of the transplanted cells was examined by microtubule-associated protein 2 (MAP2; Abcam). To visualize primary antibody reactivity, goat antimouse IgG conjugated with Alexa-Fluor 488 or 594 (Molecular Probes) was used. Images were taken with an Axioskop 2 plus microscope (Carl Zeiss AG). The remaining group of cell-treated (i.t. $n=4$, i.s. $n=4$ ) and control (i.t. $n=4$, i.s. $n=4$ ) rats were used for PCR analysis. The expression of rat target genes, including brain-derived neurotrophic factor $(B d n f)$, vascular endothelial growth factor A (Vegfa), nerve growth factor (Ngf), and neurotrophin 3 (Nt3/Sort1), were determined by quantitative real-time reverse transcription qPCR in a 7500 Real Time PCR System (Applied Biosystems, Foster City, CA, USA) using TaQMan Gene Expression Master Mix (catalog No4392938) and TagMan Gene Expression Assays 4331182 (Rn02531 967_s1/Bdnf/, Rn01511601_ml/Vegfa/, Rn01533872_ml/ Ngf/, Rn01521847_ml/Sort1/). The qPCR was carried out in a final volume of $20 \mu \mathrm{l}$ containing $500 \mathrm{ng}$ extracted RNA. The following thermal profile was used: a single cycle of reverse transcription for 30 minutes at $50{ }^{\circ} \mathrm{C}$ and 15 minutes at $95{ }^{\circ} \mathrm{C}$ for reverse transcriptase inactivation and DNA polymerase activation, followed by 40 cycles of denaturation at $95{ }^{\circ} \mathrm{C}$ for 15 seconds and annealing and extension at $60{ }^{\circ} \mathrm{C}$ for 1 minute. The results were analyzed using the integrated 7500 System SDS Software (version 1.3.1; Life Technologies, Carlsbad, CA, USA). Each dataset was normalized with an appropriate TaqMan Endogenous control selected by NormFinder (MOMA, Aarhus, Denmark). As housekeeping genes, Actb (Rn00667869_m1) was chosen for rat target genes. All qPCR reagents were provided by Applied Biosystems. Finally, the data were recalculated to relative quantities and transformed to a $\log _{2}$ scale using the Relative Expression Software Tool (REST analysis software; Qiagen, Hilden, Germany) [28]. All numerical data are presented as mean \pm standard mean error, and the relative expression ratio of the target genes was compared and analyzed statistically using REST.

\section{Results \\ Functional analysis BBB test}

The locomotor recovery of the hind legs after balloon compression injury was evaluated weekly using the BBB test. One week after $\mathrm{SCI}$, the rats displayed one of the following: no joint movement, one or two slight joint movements, or maximally extensive one joint movement only, which corresponded to a BBB score of $0-2$. The $B B B$ score was calculated from the scores of both hind legs and the BBB score was around 0.5 on average 1 week after surgery. At 1 and 2 weeks following i.t. administration ( 2 and 3 weeks after SCI), the iPS-NP-injected rats $(n=9)$ showed a rapid and significant improvement of locomotor function in their hind legs $(7.8 \pm 0.9$ and $10.1 \pm 0.7$, respectively) compared with the salineinjected controls $(n=9) \quad(3.1 \pm 0.9$ and $6.0 \pm 1.0$, respectively) $(p<0.01)$ (Fig. 1a). However, further improvement in the $\mathrm{BBB}$ score of the i.t. cell-injected animals was not so pronounced in subsequent weeks and correlated with their functional recovery, which did not advance as rapidly as it did during the first 2 weeks after treatment. A statistically significant difference in the BBB scores of the cell-injected and saline-injected animals was seen again from week 5 to the end of the experiment $(p<0.05)$. The i.s. iPS-NPtreated group $(n=11)$, similarly to the i.t.-treated one, showed a significant improvement 1 week after treatment $(1.1 \pm 0.4) \quad(p<0.01)$ followed by a gradual continuous improvement between 3 and 7 weeks after SCI $(p<0.05)$, with the greatest improvement observed during the 8th and 9th weeks after SCI $(p<0.01)$. The final scores were $8.7 \pm 0.8$ and $6.9 \pm 0.9$ in the i.t. $(n=9)$ and i.s. $(n=9)$ control groups, respectively, and $11.7 \pm 0.4$ in the i.t. iPS-NP-treated group and $12.8 \pm 0.6$ in the i.s. iPS-NPinjected group.

\section{Plantar test}

In this test, the sensitivity of the hind paw to a noxious thermal stimulus was evaluated before SCI and every 

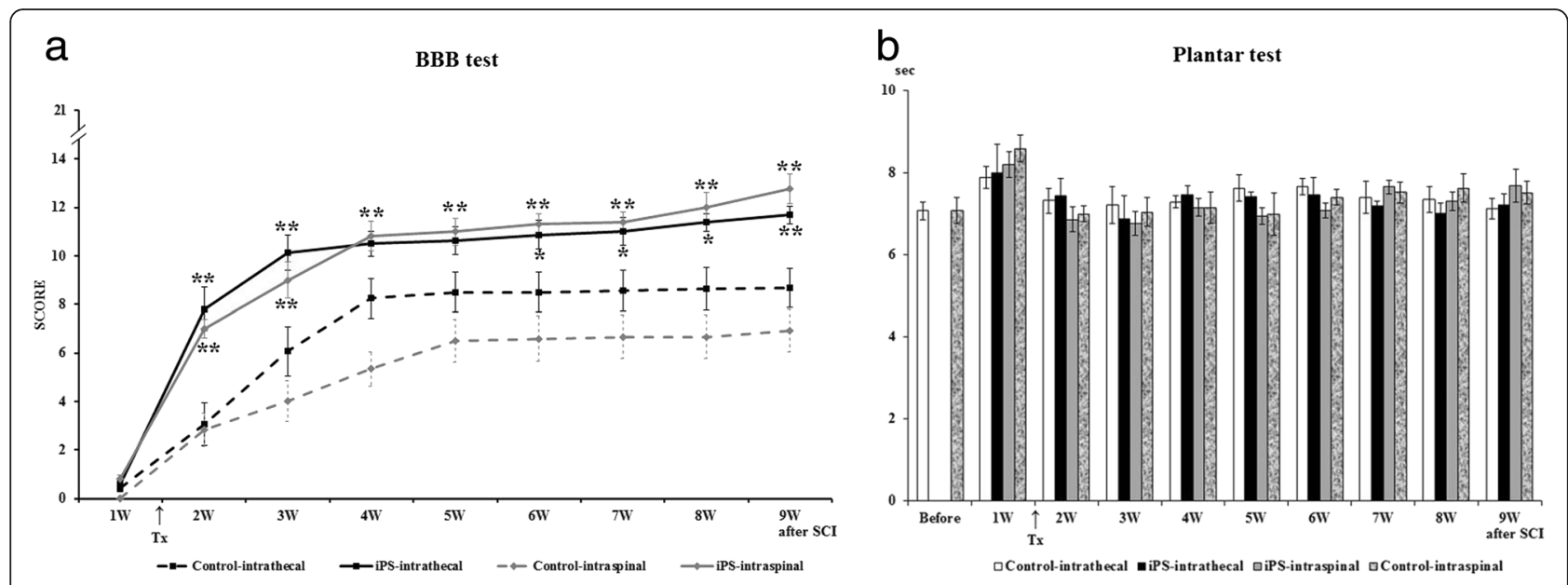

C
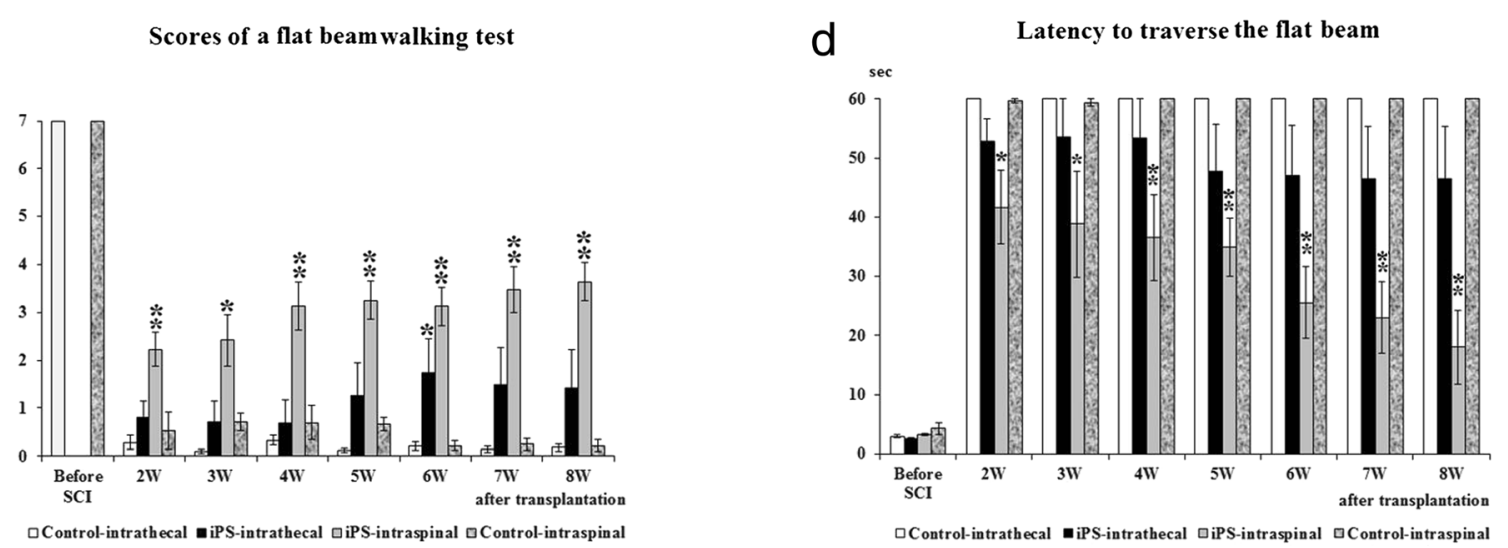

Fig. 1 Functional motor recovery evaluated by the BBB test $\mathbf{a}$. Statistical differences between the appropriate control and cell-treated groups are indicated by ${ }^{*} p<0.05$ and ${ }^{* *} p<0.01$. The sensitivity of the hind paws to thermal stimuli was examined by the Plantar test $\mathbf{b}$. The withdrawal latency before surgery is represented by the two left-most columns in the figure. There were no significant differences between the controls and the iPS-NP-treated groups throughout the experiment. The flat beam scores evaluate the locomotor function of the hind legs $\mathbf{c}$. The maximum score is 7 in a healthy animal. The differences between the i.s. control and cell-treated groups were always significant $*^{* *} p<0.01$ above the gray columns). Locomotor ability to traverse a beam with a flat surface $\mathbf{d}$. The latency decreased week by week in the treated groups when compared with the control groups. Statistical differences between the appropriate control and cell-treated groups are indicated: ${ }^{*} p<0.05$ and ${ }^{* *} p<0.01$. BBB Basso, Beattie, and Bresnahan, iPS induced pluripotent stem cell, SCl spinal cord injury, $W$ weeks, Tx day of transplantation

week after SCI (Fig. 1b). The withdrawal latency was approximately 7.1 seconds before SCI. The latency in responding to the stimulus was slightly prolonged after surgery in the i.t. control and iPS-NP-treated groups. Their withdrawal latencies gradually returned to the intact values, but were very unstable in subsequent measurement. The final observed latencies were 7.1 \pm 0.3 and $7.5 \pm 0.3$ seconds in the i.t. and i.s. control groups, respectively, $7.2 \pm 0.3$ seconds in the i.t. iPSNP-treated group, and $6.5 \pm 0.2$ seconds in the i.s. iPS-NP-treated group. There were no significant differences between the control and cell-treated groups during the 9 -week observation period $(p>0.05)$. No animals in any group showed any hypersensitivity or allodynia throughout the experiments.

\section{Beam walking test}

The locomotor performance of the hind legs while traversing the beam was evaluated using a $0-7$ point scale modified from Metz and Whishaw [27] before surgery and every week from the second week after treatment. The rats generally tried to traverse the beam during the first trial after the induction of the spinal cord lesion. When they tried to move forwards to reach the escape box, they fell off the beam because of a lack of locomotor function in their hind legs. If they could not improve their locomotor function during subsequent experiments, they appeared to become discouraged by their repeated failures and their fear of falling. They gave up trying to traverse the beam and instead remained at the same point on the beam where they were initially 
placed. This behavior was clearly seen in all control rats, independent of the route of saline injection. The scores at the beginning of the series of measurements after SCI were therefore better than those observed in the later measurements (Fig. 1c). In contrast, the rats that received cell treatment maintained their efforts to perform the task and displayed gradual functional recovery. A statistically significant difference was detected between the i.t. iPS-NP-treated and control groups $(p>0.05)$ in week 6 , while in weeks 5,7 , and 8 a strong trend towards improvement was observed. The final scores were $1.4 \pm 0.8$ out of 7 (the maximum for intact animals) in the treated group and $0.2 \pm 0.1$ in the control group. The scores in the i.s. iPS-NP-treated animals were always higher than those in the control group throughout the experiments $(p<0.01)$.

Simultaneously, the latency to traverse a beam with a flat surface was examined before SCI and every week from the second week after treatment. Before surgery, animals could easily traverse the beam in $3.0 \pm 0.2$ seconds on average (Fig. 1d). After lesioning, they lost their ability to traverse the beam using their hind legs and mainly stayed at the same point of the beam where they were placed. When they tried to move forwards to reach the escape box that was placed at the opposite end of the beam, they generally fell off the beam. This situation continued throughout the experiments in both groups of control rats. In contrast, the rats which received an i.t. injection of iPS-NPs improved their ability to traverse the beam week by week, but this improvement was not statistically significant $(p>0.05)$. Their final latency was $46.4 \pm 8.9$ seconds at 8 weeks after treatment ( 9 weeks after $\mathrm{SCI}$ ). The animals with i.s. treatment started to traverse the beam as early as 2 weeks after treatment, and their latency continuously decreased during the 8 weeks of observation. There were significant differences between the i.s. control and iPS-NP-treated groups between 2 weeks and 3 weeks after treatment $(p<0.05)$ and between 4 weeks and 8 weeks after treatment $(p<0.01)$. At the end of the series of experiments, two out of eight rats could completely traverse the beam in the i.t. iPS-NP-treated group, while seven out of eight rats could reach the opposite end of the beam in the i.s. iPS-NP-treated group. The final latency was $18.0 \pm 6.2$ seconds in the i.s. iPS-NP-treated animals. However, when comparing only those rats from both cell-treated groups that were able to accomplish the task, there were no differences in latencies between the i.t. and i.s. celltreated animals.

\section{Rotarod test}

Motor coordination was assessed using the rotarod test before surgery at a speed of $10 \mathrm{rpm}$ and every 2 weeks after treatment at a speed of $5 \mathrm{rpm}$. After surgery, the animals lost their ability to walk on the rotating rod. The time spent on the rod until falling off onto the floor plate was $3.2 \pm 0.4$ and $3.3 \pm 0.5$ seconds in the two control groups, $4.6 \pm 1.0$ seconds in the i.t. iPS-NP-treated group, and $11.7 \pm 4.8$ seconds in the i.s. iPS-NP-treated group $(n=8)$ at 2 weeks after treatment ( 3 weeks after $\mathrm{SCI})$. Similar to their performance in the beam test, the animals actively tried to keep their bodies on the rod using their front legs, which prevented them from dropping as they were able to grasp groves in the rod with their nails during the first trials after treatment. As the control rats could not recover locomotor function in their hind legs, their latency gradually decreased in subsequent measurements (Additional file 1: Figure S1). Conversely, the i.t. iPS-NP-treated group increased their time spent on the rod using their gradually recovering hind legs, although no significant differences between the i.t. control and iPS-NP-treated groups were observed throughout the experiment $(p>0.05)$. Similar results were obtained for the i.s. iPS-NP-treated animals, although these animals did improve their performance in the rotarod test week by week and performed better than the i.t.-treated animals. However, no statistically significant differences were found between any of the groups $(p>0.05)$.

\section{Cell survival and differentiation}

$\mathrm{HuNu}$-positive or MTCO2-positive grafted iPS-NPs were found at 8 weeks after transplantation in the i.s.-treated rats (Fig. 2). The surviving grafted cells, which were detected by their expression of the human mitochondrial marker MTCO2 and recognized as a mass of bright green fluorescence, accumulated in large number at the injection site with no tumor or tumor-like formation (Fig. 2a). Grafted human iPS-NPs were also detected by the human-specific nuclei protein $\mathrm{HuNu}$, identified as green nuclei and surrounded by red cytoplasm positive for the neuronal marker MAP2 (Fig. 2b). Neither HuNu-positive nor MTCO2-positive cells were identified on the surface of the pia matter or in the host parenchyma of the spinal cord in the intrathecally transplanted animals.

\section{Morphometric measurements}

Eight weeks after treatment (9 weeks after SCI), the experiments were terminated and the cross-sectional area of spared white and gray matter was measured from a $2-\mathrm{cm}$ long spinal cord section, excised between $1 \mathrm{~cm}$ cranial and $1 \mathrm{~cm}$ caudal to the injury epicenter, which was recognized as the smallest area of the spinal cord. The cross-sectional areas $\left(\mathrm{mm}^{2}\right)$ were plotted at $1 \mathrm{~mm}$ increments from the injury epicenter, which is indicated by 0 in Fig. 3a, b. When the total volume of the whole length of the extracted spinal cord segment was compared between 


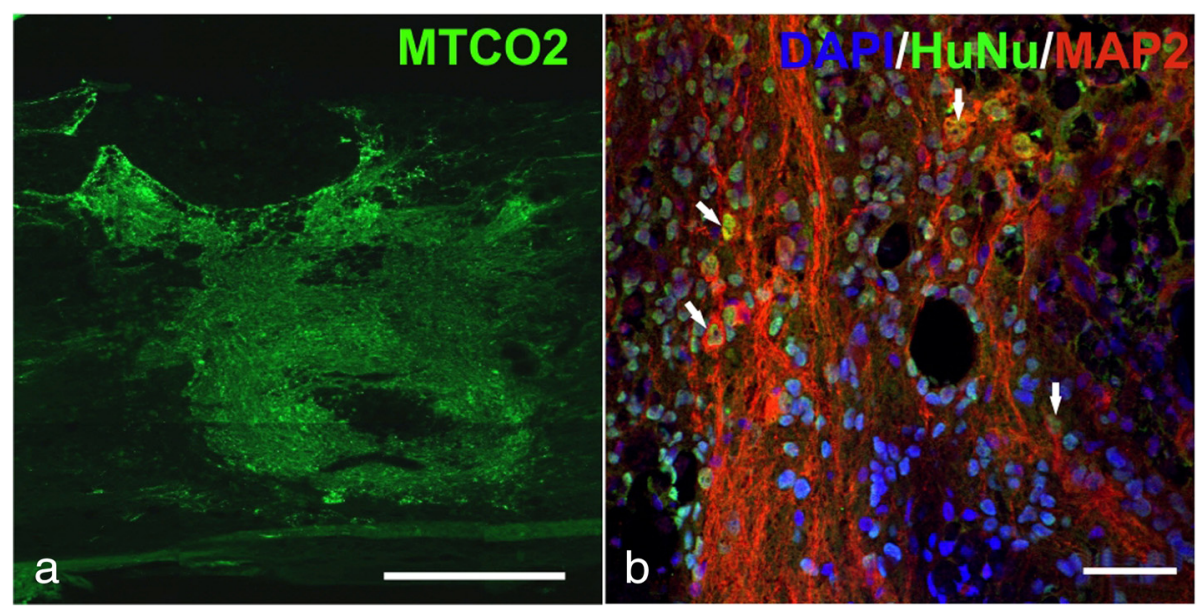

Fig. 2 MTCO2-positive transplanted cells in the host spinal cord 8 weeks after i.s. implantation of iPS-NPs. Scale bar $=500 \mu m$ a. HuNu-positive transplanted cells (green) express the neuronal marker MAP2 (red). Nuclei are stained by DAPI (blue). Colocalization is marked by arrows. Scale bar $=50 \mu \mathrm{m}$ b. DAPI 4',6-diamidino-2-phenylindole, HuNu human nuclei, MAP2 microtubule-associated protein 2

the i.s. and i.t. control groups, significant differences were apparent in the extent of white and gray matter sparing $(p<0.01)$ (Fig. 3c). In the animals that received i.t. injections (control group, $n=5$; transplanted group, $n=5$ ), a significant difference was found in the cross-sectional area of the white matter at 2 and $4 \mathrm{~mm}$ caudal to the injury epicenter $(p<0.05)$, while there was no statistical difference in the gray matter between the two groups $(p>0.05)$ (Fig. 3a). The intraspinally grafted rats $(n=5)$ showed that the white matter was spared more than in the control animals $(n=7)$ throughout the whole length of the 2-mmlong spinal segment, including at the injury epicenter $(p<0.05)$, and that the gray matter was significantly spared mainly in the region between $3 \mathrm{~mm}$ cranial and $6 \mathrm{~mm}$ caudal to the injury epicenter, where the iPS-NPs had been implanted $(p<0.05)$ (Fig. 3b). Differences also appeared in the total volume of gray matter after cell treatment, since the i.t. cell-treated spinal cord tissue was spared to a greater extent than the i.s. cell-treated tissue (Fig. 3d).

\section{In vivo tissue analysis}

SCI-induced astrogliosis is an important process in glial scar formation, as reactive astrocytes increase their expression of GFAP. The size of the GFAP-positive area was determined from serial cross-sections at $1 \mathrm{~mm}$ increments from the injury epicenter 2 months after i.t. or i.s. treatment. The results were separated into cranial (3-7 $\mathrm{mm}$ cranial to the injury epicenter), central (between $2 \mathrm{~mm}$ cranial and $2 \mathrm{~mm}$ caudal to the injury epicenter), and caudal (3-7 $\mathrm{mm}$ caudal to the injury epicenter) areas. The i.t. injection of iPS-NPs did not exert any effects against scar formation. There was no significant difference between the i.t.-injected groups in any of the investigated areas $(p>0.05)$ (Fig. 4a). In the i.s. iPS-NP-treated group, on the other hand, astrogliosis was significantly reduced in the cranial area when comparing the i.s.-treated and control groups $(p<0.05)$ (Fig. 4a).

To evaluate the neurotrophic effects of the treatment, the gene expression of rat neurotrophic factors, including BDNF, VEGF, nerve growth factor (NGF), and neurotrophin 3 (NT3), was analyzed by qPCR at 8 weeks after treatment (9 weeks after SCI). There was a significant downregulation of BDNF, VEGF, and NGF gene expression $(p<0.05)$ and no effect on NT3 expression $(p>0.05)$ in the i.t. iPS-NP-treated group compared with the control group (Fig. 4b). In the intraspinally treated animals, the gene expression of BDNF, VEGF, and NGF was higher than that of NT3 and lower than in the control animals, but statistically significant differences were not found between the i.s. iPSNP-treated and saline-injected group $(p>0.05)$.

The elevated expression of GAP43 leads to axonal sprouting in the spinal cord [23]. To assess axonal regeneration and sprouting, the number of GAP43-positive fibers was counted from serial cross-sections. If the number of GAP43-positive fibers in the control group is considered as $100 \%$, the number of positive fibers increased by $168.3 \pm 9.1 \%$ in the i.t. iPS-NP-treated group and $288.7 \pm$ $66.5 \%$ in the i.s. iPS-NP-treated group (Fig. 4c). GAP43positive fibers were seen more abundantly in the i.t. celltreated group than in the saline-treated control group, and even more GAP43-positive fibers were present in the i.s. cell-treated animals.

\section{Discussion}

Our study, for the first time, evaluated the i.t. application of iPS-NPs into a rat model of SCI. We have shown that 

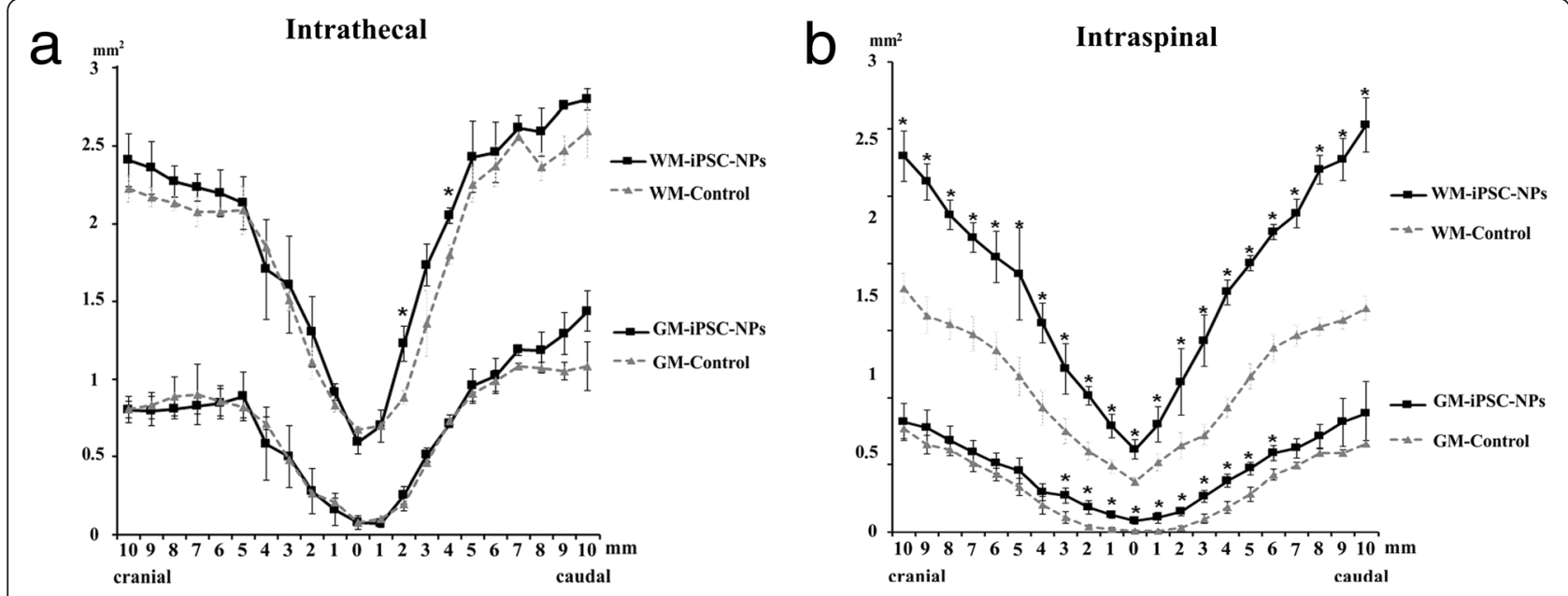

C

Total volume
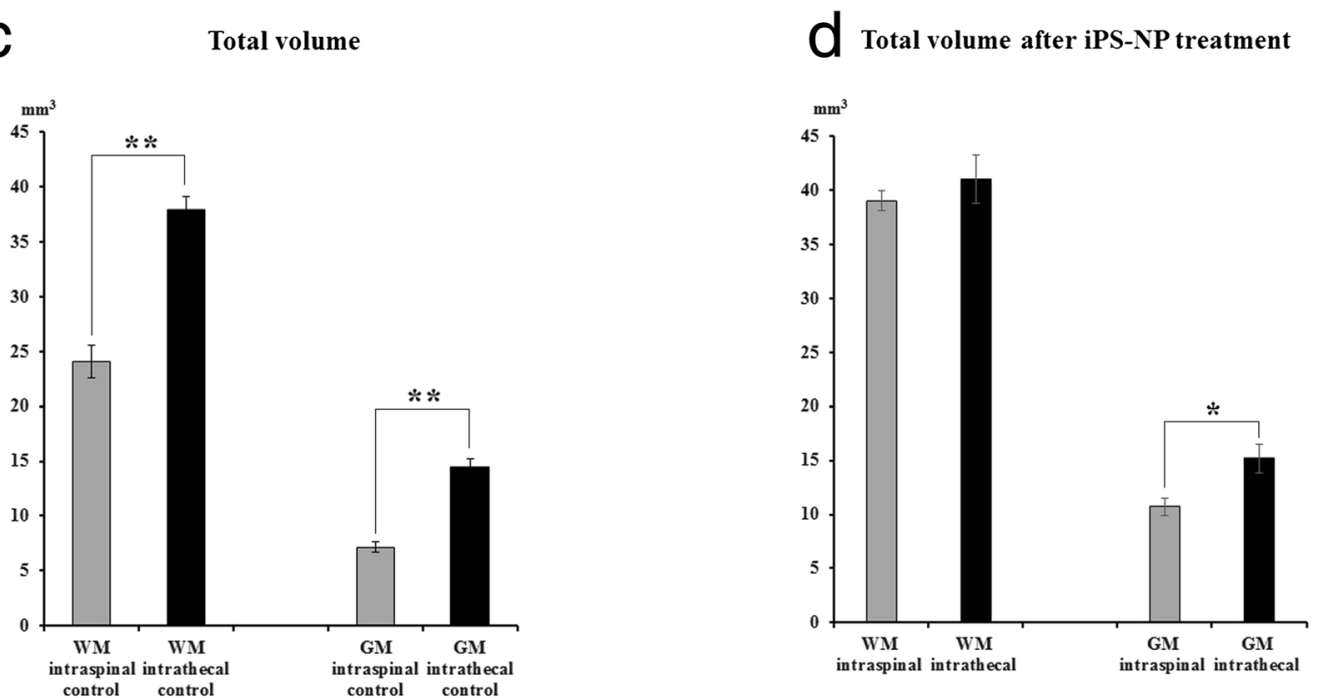

Fig. 3 Morphometric measurement of the white matter $(W M)$ and gray matter $(G M)$. The cross-sectional area $\left(\mathrm{mm}^{2}\right)$ is plotted at $1 \mathrm{~mm}$ increments in both the cranial and caudal directions from the injury epicenter, which is indicated by the number 0 . The WM was significantly spared only 2 and $4 \mathrm{~mm}$ caudal to the injury epicenter in the i.t-treated group a. In contrast, the WM and GM were prominently spared in the i.s. iPS-NP-transplanted group $\mathbf{b}$. The total volume of a 2 -mm-long spinal segment, including the injury epicenter, is compared between the i.s. and i.t. control groups $\mathbf{c}$. Statistically significant differences were found in both the volume of the WM and also the volume of the GM $(* * 0.01)$. Total volume of a 2-mm-long spinal segment after iPS-NP treatment $\mathbf{d}$. There is a significant difference in the volume of the GM between i.s. and i.t. administration $\left({ }^{*} p<0.05\right)$. iPSC-NP neural precursors derived from induced pluripotent stem cells

the i.t. injection of iPS-NPs can improve functional locomotor outcome as determined not only by BBB analysis, but also, to some extent, by the flat beam test. This implies that the i.t.-treated animals could recover the ability to support their body weight by using their hind legs, but more sophisticated and complex functions, such as coordination between the forelimbs and hindlimbs, more precise paw placement, and maintaining their balance by using their legs, recovered fully in only $25 \%$ of the i.t. cell-treated animals. On the other hand, the i.s. injection of iPS-NPs improved the functional outcome in all of the locomotor tests. In agreement with our previous findings, intraspinally injected iPS-NPs survived well for the whole observation period [9]. We could not trace any surviving grafted cells using human nuclei and mitochondria markers $(\mathrm{HuNu}$, MTCO2) 8 weeks after i.t. injection. The inability to detect surviving cells may be owing to the fact that these transplanted cells were widely dispersed in the CSF or that the conditions in the CSF were unfavorable for the long-term survival of the transplanted cells. However, cell survival after i.t. application could be dependent on the cell type used and/or on the time window in which the cells were administered. Mothe et al. [29] compared 


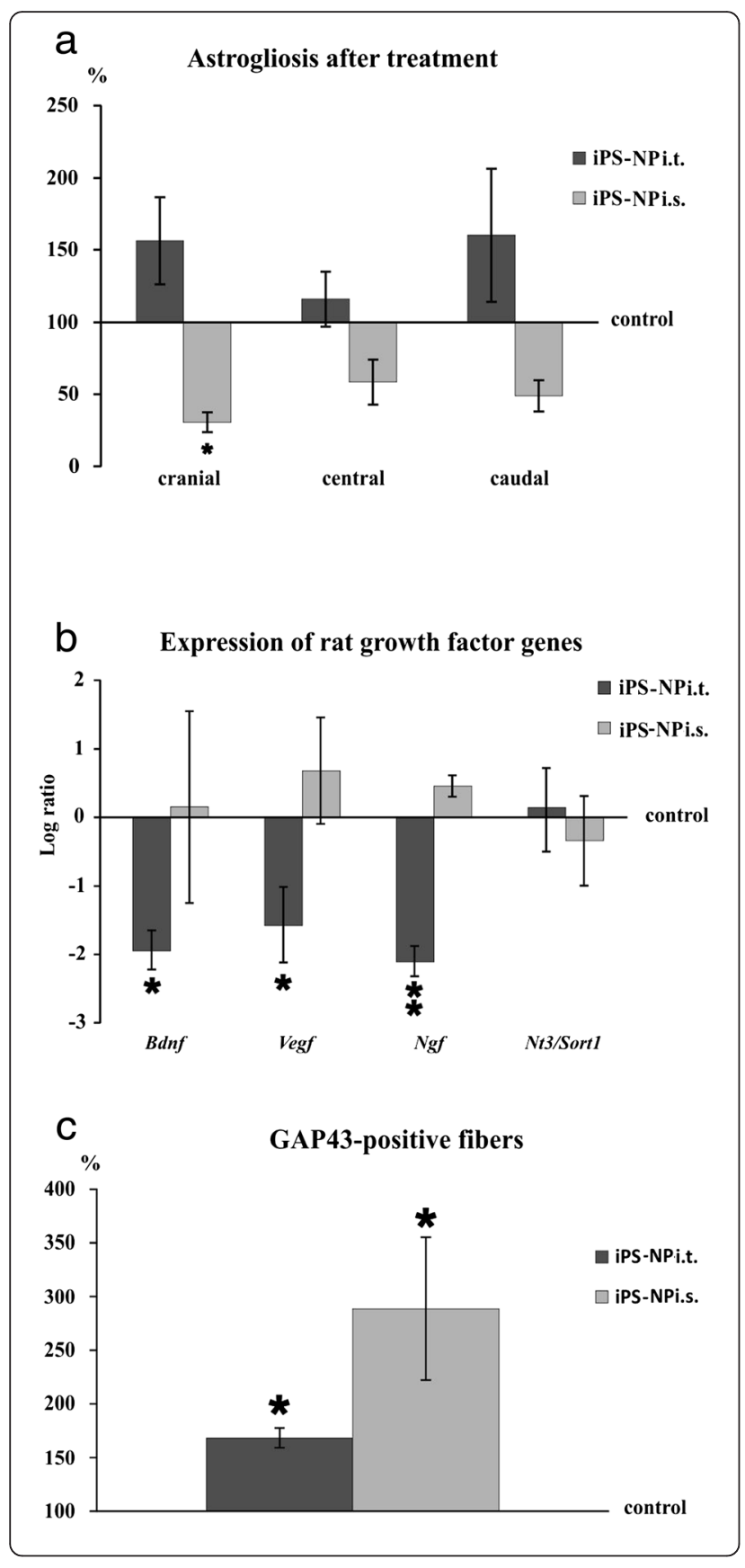

Fig. 4 GFAP-positive areas are compared in three different parts of the spinal segment: cranial, between 3 and $7 \mathrm{~mm}$ cranial; central, between $2 \mathrm{~mm}$ cranial and $2 \mathrm{~mm}$ caudal; and caudal, between 3 and $7 \mathrm{~mm}$ caudal to the injury epicenter $\mathbf{a}$. The average GFAP-positive area around the lesion in the appropriate control group is shown as the baseline of $100 \%$. Rat gene expression of BDNF, VEGF, nerve growth factor (NGF), and neurotrophin 3 (NT3) analyzed by qPCR $\mathbf{b}$. The level of the control expression is indicated by the number 0 . An increase or decrease from this level is shown as positive or negative scale numbers, respectively. The levels of BDNF, VEGF, and NGF in the i.t. iPS-NP-treated group are significantly lower than those in the control animals. There was no significant difference between the i.s. iPS-NP-treated and control groups in terms of gene expression. Axonal sprouting evaluated by GAP43-positive fibers $\mathbf{c}$. The average number of positive fibers in the control group is shown as the baseline of $100 \%$. Both cell-treated groups show the increased expression of GAP43, which was more pronounced in the i.s. cell-treated animals. iPS-NP neural precursors derived from induced pluripotent stem cells, i.s. intraspinal, i.t. intrathecal

intrathecally transplanted neural stem/progenitor cells (NSPCs) and bone marrow stromal cells (BMSCs) in a clip model of SCI. By four weeks after transplantation, more NSPCs had migrated to the lesion site relative to BMSCs and uninjured animals. However, there was no preferential homing of either of these types of cells into the parenchyma of the injury site, and most of the transplanted cells remained in the i.t. space. By 4 weeks post transplantation, significantly more NSPCs were found at the site of injury (T8), while the majority of BMSCs were found at the injection site (L3-L5). Similarly, when MSCs were transplanted intrathecally at the lesion site by a catheter guided from the cisterna magna 1 week after a balloon compression lesion, the majority of the transplanted cells were seen attached to the pia mater 28 days after SCI, with only a few cells having penetrated into the white matter [30]. We reported previously only a few human MSCs attached to the dorsal surface of the lesioned spinal cord 2 weeks after i.t. application with no survival of cells 8 weeks after application. However, we detected white matter sparing, remodeling of the glial scar, and a decrease in the levels of inflammatory cytokines in the center of the lesion along with enhanced locomotor function [31].

The morphometric measurements revealed that tissue sparing was not as marked in the white and gray matter after i.t. treatment compared with the intraspinally injected animals. Significant tissue sparing was observed in the area of the spinal cord caudal, but not cranial, to the injury epicenter after treatment. This could be explained by the fact that transplanted cells which were injected in the lumbar area may not have been able to reach beyond the balloon-compressed site because the subarachnoid space may have narrowed or collapsed as SCI damage progressed. The direct injection of iPS-NPs is likely to cause tissue damage around the injection site. 
The volumes of white and gray matter were reduced more in the i.s.-injected controls than in the controls with an i.t. injection, indicating further damage due to the more invasive surgical procedure. Moreover, the volume of the gray matter was spared significantly more in the i.t.-treated group than in the i.s.-treated group. This finding corresponded to lower BBB scores in i.s. controls than in i.t. controls; however, this result was not statistically significant. The beneficial effect of i.s. application might be influenced by the fact that saline-injected i.s. animals scored worse in the BBB test and their spinal cord tissue was more damaged than in i.t.-injected control rats. It is worth mentioning that the tissue cavity which develops after lesioning was reduced or partially filled with grafted cells after i.s. treatment, but i.t.injected cells were not able to home into the lesion. The volumes of the white and gray matter in i.s. cell-injected animals were analyzed and calculated from a segment of the spinal cord that included transplanted cells, since grafted iPS-NPs incorporated nicely into the host tissue. However, it was impossible to identify transplanted cells in the cross-sections stained with Luxol-Fast Blue and Cresyl Violet, which were used to distinguish the white and gray matter.

Axonal regeneration was facilitated by i.t.-injected cell treatment, but was not accompanied by the upregulation of growth factors in the host tissue of the spinal cord 2 months after grafting (Fig. 4c). Rat neurotrophic factors, including BDNF, VEGF, NGF, and NT3, did not significantly change after i.s. injection of iPS-NPs compared with those in the control animals. However, we observed previously the upregulated expression of human neurotrophin genes for NGF, FGF8, and GDNF [9]. Improved axonal sprouting was therefore most probably supported by exogenous neurotrophic factors rather than host neurotrophins. However, our analysis was performed at the end of the study. It is likely that a transient upregulation of host neurotrophic factors might be observed at earlier time points after cell application.

Direct injection of iPS-NPs into the host tissue has been shown to be effective not only in a model of SCI [9], but also in the middle cerebral artery occlusion model of stroke [22]. In both studies, the grafted cells differentiated into tissue-specific neurons (striatal dopaminergic neurons or interneurons and motoneurons); and in both settings, the injection of iPS-NPs had a dual effect that resulted in rapid functional improvement followed by slow cell differentiation and partial reconstruction of the impaired pathways. Similarly, in the studies by Lu et al. $[32,33]$ intraspinally transplanted human iPS-derived neural stem cells successfully extended large numbers of axons even over long distances and formed synapses with host neurons after complete spinal transection or hemisection. In our study, it seems that the transplanted cells could not directly reach the lesioned spinal cord via the i.t. route, and therefore this route of administration was not as effective as the i.s. route. However, there was a paracrine effect of iPS-NPs that did not require the presence of cells in the tissue. This paracrine effect was strong enough to improve functional outcome and axonal sprouting and to partially spare the damaged tissue. The obtained results are comparable with the effect of i.t.-injected MSCs and NSPCs [29, 31]. Cells dispersed in the CSF will be less likely to make the contacts necessary for tumor or teratoma formation, which has to be taken into consideration for i.s. application.

\section{Conclusion}

From the results of the present study, it is evident that i.t. application of iPS-NPs had a moderate therapeutic influence (resulting in locomotor improvement, moderate tissue sparing, and axonal sprouting) on SCI through a paracrine mechanism that does not require the presence of cells in the tissue. This effect was comparable with that of other studies reporting similar results after i.t. treatment using MSCs or NSPCs in SCI. Further studies are still required to answer the question of whether repeated i.t. injections and/or an increase in the number of cells transplanted can exert even a more beneficial effect in the treatment of SCI.

\section{Additional file}

Additional file 1: Figure S1. Showing locomotor coordination evaluated by the rotarod test. The time spent on the rod gradually increased in both treated groups, but there were no statistically significant differences between the control and treated groups. (JPG $42 \mathrm{~kb}$ )

\begin{abstract}
Abbreviations
BBB: Basso, Beattie, and Bresnahan; BDNF: Brain-derived neurotrophic factor; bFGF: Basic Fibroblast growth factor; BMSC: Bone marrow stromal cell; CSF: Cerebrospinal fluid; DMEM: Dulbecco's modified Eagle's medium; EGF: Epidermal growth factor; FGF: Fibroblast growth factor; GAP: Growthassociated protein; GDNF: Glia-derived neurotrophic factor; GFAP: Glial fibrillary acidic protein; HuNu: Human nuclei; i.s.: Intraspinal; i.t.: Intrathecal; IL: Interleukin; iPS: Induced pluripotent stem cell; iPS-NP: Neural precursor derived from induced pluripotent stem cells; MAP2: Microtubule-associated protein 2; MSC: Mesenchymal stem cell; MTCO2: mitochondrially encoded cytochrome c oxidase II; NGF: Nerve growth factor; NSPC: Neural stem/progenitor cell; NT3: Neurotrophin 3; NT4: Neurotrophin 4; SCl: Spinal cord injury; T8: Thoracic vertebra 8; VEGF: Vascular endothelial growth factor.
\end{abstract}

Competing interests

The authors declare that they have no competing interests.

Authors' contributions

TA carried out the surgeries, cell transplantation, behavioral testing, and manuscript drafting. NR carried out the cell preparation and differentiation, and immunohistochemistry, and helped to draft the manuscript. JR participated in the histological morphometric analyses, performed the statistical analysis, and helped to draft the manuscript. MJ-U participated in the design of the study and helped to draft the manuscript. ES participated in the design of the study and helped to draft the manuscript. PJ conceived of the study, participated in its design and coordination, and helped to draft the manuscript. All authors read and approved the final manuscript. 


\section{Acknowledgements}

This study was supported by a grant from MEYS Kontakt II LH12024. The authors thank James Dutt for critical reading of the manuscript.

\section{Author details}

${ }^{1}$ Department of Neuroscience, Institute of Experimental Medicine, Academy of Sciences of the Czech Republic, Videnska 1083, 14220 Prague 4, Czech Republic. ${ }^{2}$ Department of Neuroscience, 2nd Faculty of Medicine, Charles University, Plzenska 130/221, 15000 Prague 5, Czech Republic. ${ }^{3}$ Department of Neurosurgery, New York Medical College, Valhalla, NY 10595, USA.

Received: 19 October 2015 Revised: 11 November 2015 Accepted: 2 December 2015 Published online: 22 December 2015

\section{References}

1. Angelos MG, Kaufman DS. Pluripotent stem cell applications for regenerative medicine. Curr Opin Organ Transplant. 2015;20:663-70.

2. Erceg S, Lukovic D, Moreno-Manzano V, Stojkovic M, Bhattacharya SS. Derivation of cerebellar neurons from human pluripotent stem cells. Curr Protoc Stem Cell Biol. 2012; Chapter 1:Unit 1H 5.

3. Hodgetts SI, Edel M, Harvey AR. The state of play with iPSCS and spinal cord injury models. J Clin Med. 2015;4:193-203.

4. Jin X, Lin T, Xu Y. Stem cell therapy and immunological rejection in animal models. Curr Mol Pharmacol. 2015. doi:10.2174/1874467208666150928153511.

5. Lee-Kubli CA, Lu P. Induced pluripotent stem cell-derived neural stem cell therapies for spinal cord injury. Neural Regen Res. 2015;10:10-6.

6. Fujimoto Y, Abematsu M, Falk A, Tsujimura K, Sanosaka T, Juliandi B, et al. Treatment of a mouse model of spinal cord injury by transplantation of human induced pluripotent stem cell-derived long-term self-renewing neuroepithelial-like stem cells. Stem Cells. 2012;30:1163-73.

7. Nori S, Okada Y, Yasuda A, Tsuji O, Takahashi Y, Kobayashi Y, et al. Grafted human-induced pluripotent stem-cell-derived neurospheres promote motor functional recovery after spinal cord injury in mice. Proc Natl Acad Sci USA. 2011;108:16825-30.

8. Oh J, Lee Kl, Kim HT, You Y, Yoon do H, Song KY, et al. Human-induced pluripotent stem cells generated from intervertebral disc cells improve neurologic functions in spinal cord injury. Stem Cell Res Ther. 2015;6:125.

9. Romanyuk N, Amemori T, Turnovcova K, Prochazka P, Onteniente B, Sykova E, et al. Beneficial effect of human induced pluripotent stem cell-derived neural precursors in spinal cord injury repair. Cell Transplant. 2015;24:1781-97.

10. Sareen D, Gowing G, Sahabian A, Staggenborg K, Paradis R, Avalos P, et al. Human induced pluripotent stem cells are a novel source of neural progenitor cells (iNPCs) that migrate and integrate in the rodent spinal cord. J Comp Neurol. 2014;522:2707-28.

11. All AH, Gharibani P, Gupta S, Bazley FA, Pashai N, Chou BK, et al. Early intervention for spinal cord injury with human induced pluripotent stem cells oligodendrocyte progenitors. PLoS One. 2015;10:e0116933.

12. Salewski RP, Mitchell RA, Li L, Shen C, Milekovskaia M, Nagy A, et al. Transplantation of induced pluripotent stem cell-derived neural stem cells mediate functional recovery following thoracic spinal cord injury through remyelination of axons. Stem Cells Transl Med. 2015;4:743-54

13. Pajer K, Nemes C, Berzsenyi S, Kovacs KA, Pirity MK, Pajenda G, et al. Grafted murine induced pluripotent stem cells prevent death of injured rat motoneurons otherwise destined to die. Exp Neurol. 2015;269:188-201.

14. Tsuji O, Miura K, Okada Y, Fujiyoshi K, Mukaino M, Nagoshi N, et al. Therapeutic potential of appropriately evaluated safe-induced pluripotent stem cells for spinal cord injury. Proc Natl Acad Sci U S A. 2010;107:12704-9.

15. Bakshi A, Barshinger AL, Swanger SA, Madhavani V, Shumsky JS, Neuhuber $B$, et al. Lumbar puncture delivery of bone marrow stromal cells in spinal cord contusion: a novel method for minimally invasive cell transplantation. J Neurotrauma. 2006;23:55-65.

16. Judas Gl, Ferreira SG, Simas R, Sannomiya P, Benicio A, da Silva LF, et al. Intrathecal injection of human umbilical cord blood stem cells attenuates spinal cord ischaemic compromise in rats. Interact Cardiovasc Thorac Surg. 2014;18:757-62.

17. Paul C, Samdani AF, Betz RR, Fischer I, Neuhuber B. Grafting of human bone marrow stromal cells into spinal cord injury: a comparison of delivery methods. Spine. 2009;34:328-34.

18. Volarevic V, Erceg S, Bhattacharya SS, Stojkovic P, Horner P, Stojkovic M. Stem cell-based therapy for spinal cord injury. Cell Transplant. 2013;22:1309-23.
19. Amemori T, Jendelova P, Ruzickova K, Arboleda D, Sykova E. Cotransplantation of olfactory ensheathing glia and mesenchymal stromal cells does not have synergistic effects after spinal cord injury in the rat. Cytotherapy. 2010;12:212-25.

20. Arboleda D, Forostyak S, Jendelova P, Marekova D, Amemori T, Pivonkova H, et al. Transplantation of predifferentiated adipose-derived stromal cells for the treatment of spinal cord injury. Cell Mol Neurobiol. 2011;31:1113-22.

21. Amemori T, Romanyuk N, Jendelova P, Herynek V, Turnovcova K, Prochazka P, et al. Human conditionally immortalized neural stem cells improve locomotor function after spinal cord injury in the rat. Stem Cell Res Ther. 2013;4:68.

22. Polentes J, Jendelova P, Cailleret M, Braun H, Romanyuk N, Tropel P, et al. Human induced pluripotent stem cells improve stroke outcome and reduce secondary degeneration in the recipient brain. Cell Transplant. 2012;21:2587-602.

23. Bomze HM, Bulsara KR, Iskandar BJ, Caroni P, Skene JH. Spinal axon regeneration evoked by replacing two growth cone proteins in adult neurons. Nat Neurosci. 2001;4:38-43.

24. da Costa ES, Carvalho AL, Martinez AM, De-Ary-Pires B, Pires-Neto MA de Ary-Pires R. Strapping the spinal cord: an innovative experimental model of CNS injury in rats. J Neurosci Methods. 2008;170:130-9.

25. Vanicky I, Urdzikova L, Saganova K, Cizkova D, Galik J. A simple and reproducible model of spinal cord injury induced by epidural balloon inflation in the rat. J Neurotrauma. 2001;18:1399-407.

26. Basso DM, Beattie MS, Bresnahan JC. A sensitive and reliable locomotor rating scale for open field testing in rats. J Neurotrauma. 1995;12:1-21.

27. Metz GA, Whishaw IQ. The ladder rung walking task: a scoring system and its practical application. J Vis Exp. 2009;28;1-4, doi: 10.3791/1204.

28. Pfaffl MW, Horgan GW, Dempfle L. Relative expression software tool (REST) for group-wise comparison and statistical analysis of relative expression results in real-time PCR. Nucleic Acids Res. 2002;30:e36.

29. Mothe AJ, Bozkurt G, Catapano J, Zabojova J, Wang X, Keating A, et al. Intrathecal transplantation of stem cells by lumbar puncture for thoracic spinal cord injury in the rat. Spinal Cord. 2011;49:967-73.

30. Cizkova D, Novotna I, Slovinska L, Vanicky I, Jergova S, Rosocha J, et al Repetitive intrathecal catheter delivery of bone marrow mesenchymal stromal cells improves functional recovery in a rat model of contusive spinal cord injury. J Neurotrauma. 2011;28:1951-61.

31. Urdzikova LM, Ruzicka J, LaBagnara M, Karova K, Kubinova S, Jirakova K, et al Human mesenchymal stem cells modulate inflammatory cytokines after spinal cord injury in rat. Int J Mol Sci. 2014;15:11275-93.

32. Lu P, Wang Y, Graham L, McHale K, Gao M, Wu D, et al. Long-distance growth and connectivity of neural stem cells after severe spinal cord injury. Cell. 2012;150:1264-73.

33. Lu P, Woodruff G, Wang Y, Graham L, Hunt M, Wu D, et al. Long-distance axonal growth from human induced pluripotent stem cells after spinal cord injury. Neuron. 2014;83:789-96.

\section{Submit your next manuscript to BioMed Central and we will help you at every step:}

- We accept pre-submission inquiries

- Our selector tool helps you to find the most relevant journal

- We provide round the clock customer support

- Convenient online submission

- Thorough peer review

- Inclusion in PubMed and all major indexing services

- Maximum visibility for your research

Submit your manuscript at www.biomedcentral.com/submit 